\title{
An Empirical Study on Intercultural Sensitivity of Foreign Language Majors and Related Influencing Factors
}

\author{
Meina Feng \\ Zhejiang Yuexiu University, Shaoxing, China \\ Email: 20131050@zyufl.edu.cn
}

How to cite this paper: Feng, M.N. (2021) An Empirical Study on Intercultural Sensitivity of Foreign Language Majors and Related Influencing Factors. Open Access Library Journal, 8: e7833.

https://doi.org/10.4236/oalib.1107833

Received: August 5, 2021

Accepted: August 31, 2021

Published: September 3, 2021

Copyright ( 2021 by author(s) and Open Access Library Inc.

This work is licensed under the Creative Commons Attribution International License (CC BY 4.0).

http://creativecommons.org/licenses/by/4.0/ (c) (i) Open Access

\begin{abstract}
This study investigated the intercultural sensitivity of 220 students who majored in foreign languages by using intercultural sensitivity scale (ISS) developed by Chen Guoming and Starosta (2000). Results showed that the intercultural sensitivity of foreign language majors is above average. In the five dimensions, respect for cultural differences is the strongest, interaction engagement is the second, followed by interaction attentiveness and interaction enjoyment, and interaction confidence is the weakest. Pearson's correlation analysis showed that interaction confidence is strongly correlated with interaction engagement and interaction enjoyment. The factors of gender, scores of CET4/CET6, overseas experience and different language major categories which may influence intercultural sensitivity were also surveyed. The results showed that there is no significant difference in intercultural sensitivity between male and female students, but there is a difference in the dimension of respect for cultural differences. And there is no significant difference in intercultural sensitivity between those who have passed CET4/CET6 or not and those who have short-term overseas experience or not. There is no significant difference among different language majors, but there is a difference in interaction confidence among them.
\end{abstract}

\section{Subject Areas}

Language Education

\section{Keywords}

Foreign Language Students, Intercultural Sensitivity, Influencing Factors 


\section{1. 研究背景}

在 “一带一路” 倡议和推进构建人类命运共同体的时代背景下, 积极推 动跨文化交流, 是国家战略的需求。它既能帮助不同民族求同存异, 增进文 化间的信任和交融, 又能为国家之间的和平合作、互利共赢奠定重要基础。 同时, 跨文化交流也是学科发展的重要任务, 大学英语教学指南(2020 版)指 出大学英语课程重要任务之一是进行跨文化教育, 语言是文化的载体, 同时 也是文化的组成部分, 学生学习和掌握英语这一交流工具, 除了学习、交流 先进的科学技术或专业信息之外, 还要了解国外的社会与文化, 增进对不同 文化的理解、对中外文化异同的意识, 培养跨文化交际能力。[1]此外, 学术 界西方学者 King \& Magolda (2005)指出: “在这个全球相互依赖日益加深的 时代, 教育的紧迫任务就是要培养具有跨文化能力的公民, 他们在面临涉及 多元文化视角的问题时, 能够做出明智的、道德的决策。” [2]中国跨文化交 际学会会长孙有中教授(2016)指出, “在中外文明全方位交流互鉴的大背景 下, 可以说当代中国比历史上任何时期都更需要具有跨文化能力的高层次国 际化人才。” [3] 综上所述, 当前跨文化交际能力人才的培养变得尤为重要和 紧迫。

\section{2. 跨文化敏感度概述}

跨文化交际能力是指不同文化背景的人们之间的交流互动。它包含三个 相互依存的层面: 认知, 即跨文化意识; 情感, 即跨文化敏感度; 行为, 即 跨文化效力。跨文化敏感度是跨文化交际能力的情感层面。Chen \& Starosta (2000)指出跨文化敏感度是个体有能力理解和欣赏不同的文化, 从而促进跨文 化交际中行为的适应性和有效性。[4]从定义可以看出, 跨文化敏感度强调交 际个体首先要有理解、欣赏和接受不同文化的积极动机, 才能在跨文化交际 过程中进行积极的互动。Bennett(1993)提出了著名的跨文化发展模型(DMIS, Developmental Model of Intercultural Sensitivity), 共包括六个阶段: (1) 否认 差异阶段 (Denial)、（2）抵制差异阶段(Defense)、（3）最小化差异阶段 (Minimizations)、(4) 认同差异阶段 (Acceptance)、(5) 适应差异阶段 (Adaptation)、(6) 差异融合阶段(Integration), 经历了这六个发展阶段, 个体 可以逐渐形成跨文化敏感度。[5]对跨文化敏感度的测定可以在很大程度上反 映出被试对象跨文化交际能力的高低。因此, 跨文化敏感度受到教育者和研 究者的广泛关注。

国外就跨文化敏感度的研究主要涵盖三个方面: 研发测量工具(Chen \& Starosta 2000 [4]; Hammer \& Bennett 2002 [6]; Hammer, Bennett \& Wiseman 2003 [7]); 分析特定人群的跨文化敏感度现状(Straffon 2003 [8]; Holma, Nokelainenb \& Tirri 2009 [9]; Tamama \& Krauss 2017 [10]); 以及研究特定环境或 特殊手段干预之下对跨文化敏感度的影响(Frit, Möllenberg \& Chen 2002 [11]; Bloom \& Miranda 2015 [12]; Kim, Choi \& Tatar 2017 [13])。国内跨文化敏感度 研究兴起于 20 世纪末, 主要包括以下几个方面: 对学生或教师跨文化敏感度 的调查(周杏英 2007 [14]; 邵思源、陈坚林, 2011 [15]; 李燕 2015 [16]; 崔海 
英、王静 2018 [17])、不同对象跨文化敏感度对比分析(彭世勇 2007 [18]; 王 晓玲 2012 [19])、跨文化敏感度和其他因素的相关性研究(贾曼丽 2014 [20]; 张芝花 2015 [21])及跨文化敏感度发展模式(DMIS)的应用等(赵萱 2011 [22]; 蒋璜 2016 [23])。对高校学生跨文化敏感度的实证调查中, 一些研究者的调查 结果显示国内高校大学生的跨文化敏感度总体处于 “中等” 或 “中等偏下” 水平(周杏英 2007 [14]; 马黎 2017 [24]; 崔海英、王静 2018 [17])。也有研究 发现文化接触多的文科专业学生跨文化敏感度水平好于理工科专业学生(彭 世勇 2007 [18]、李燕 2015 [16])。那么与文化接触多的外语专业学生的跨文化 敏感度现状如何, 这一研究对象鲜有人调查。“外语教学将语言、文化、交 际等有机结合, 掌握一种语言就是理解一种文化, 语言学习的最终目的乃是 要实现得体和有效的跨文化沟通。因此, 外语教学必然成为实施跨文化教育 最有效的阵地之一” (张红玲 2012 [25]; 孙有中 2016 [3])。而外语专业学生就 是我国推进跨文化交流和跨文化传播的主力军。本研究将重点考察外语专业 学生的跨文化敏感度现状, 探究其影响因素, 以期为外语专业学生跨文化敏 感度及跨文化交际能力的提高提供实证依据。

\section{3. 研究方法}

\section{(一) 研究问题}

本研究将探讨以下三个问题:

（1）外语专业学生跨文化敏感度水平状况如何?

（2）跨文化敏感度五个层面之间的相关性如何?

（3）性别、四六级成绩、出国经历及外语专业类别四个因素对跨文化敏 感度是否有影响?

\section{(二) 研究对象}

本研究的研究对象为某高校 2018 级 220 名外语专业大三学生, 年龄在 20 22 岁之间, 语种分别为西班牙语、德语、葡萄牙语、意大利语、法语、 俄语、捷克语。问卷调查时间为 2021 年 6 月 21 23 日, 回收有效问卷数为 220 份, 其中男生 41 人, 女生 179 人, 调查时间和研究对象的选择基于以下 考虑: 学生为大三外语专业学生, 大部分的核心课程、英语课程及跨文化课 程已修完; 调查时间为本学期的第 17 周, 学生刚刚修完了一门文化类课程。

\section{(三) 研究工具}

研究采用问卷调查的方法收集数据。问卷是 Chen \& Starosta 于 2000 年开 发的跨文化敏感度量表 ISS (Intercultural Sensitivity Scale)。它的有效性、可靠 性已得到验证。(Fritz \& Möllenberg 2002, Cronbach's $\alpha=0.88$ ) [11]。这份量表 的设计为李克特 5 级量表形式, 共有 24 个题目, 对跨文化敏感度的五个维度 的变量, 即交际参与度、差异认同感、交际信心、交际愉悦感和交际专注度 分别进行了测量。为了使受试者更好地理解量表中的 24 个题目, 研究者使用 的是由沈兴涛(2012)翻译的中文版的跨文化敏感度量表 [26]。量表中题目 1、 $11 、 13 、 21 、 22 、 23$ 和 24 用于测评交际参与度(指交际参与倾向和参与程度); 题目 2、7、8、16、18、20 用于测评差异认同感(指对文化差异的意识和认同 程度); 交际信心(指参与交际的信心)涵盖的题目为 $3 、 4 、 5 、 6 、 10$; 交际愉 
悦感感(指参与交际获得的满足和快乐)涵盖的题目为 9、12、15; 交际专注度 (指交际参与过程中交际主体的专注意愿和专注程度)涵盖的题目为 14、17、

19 。

\section{(四) 数据收集与分析}

本研究采用的是定量研究方法, 通过问卷采集数据。问卷的分析采用 SPSS23.0 软件包进行统计和分析。分析数据之前, 研究人员对跨文化敏感度 量表中的 9 个反向设计的问题(2、4、7、9、12、15、18、20、22)进行了数据 转换。然后运用描述性统计、皮尔逊相关性分析、独立样本 $\mathrm{T}$ 检验、单因素 方差等方式，完成上述三个研究问题的分析。

\section{4. 结果与分析}

\section{（一）外语专业学生跨文化敏感度水平现状分析}

被试的跨文化敏感度水平总的均值为 3.7996。他们所选的具体数字计为 该题所得分数， 5 分代表跨文化敏感度水平最高，4 5 分为中上水平， $3 \sim 4$ 分 为中等水平。由此可见, 学生的跨文化敏感度水平处于中等偏上水平。表 1 所示, 跨文化敏感度五个维度中, 差异认同感水平最高 $(M=4.1826)$, 交际参 与度 $(M=3.9182)$ 和交际专注度(3.9136)次之, 交际愉悦感 $(M=3.7576)$ 略低, 交际信心 $(M=3.1309)$ 最低。

跨文化敏感度频率分布显示, 1 名学生选择结果为 3 分以下 2 分以上, 占比 $0.45 \%$; 43 名学生选择结果为 3 3.5 分, 占比 $19.5 \%$; 113 名学生选择结 果为 4 分以下 3.5 分以上, 占比 $51.4 \% ; 68$ 名学生选择结果为 $4 \sim 5$ 分, 占比 $30.9 \%$ 。没有 2 分以下的学生。可见接近 $1 / 3$ 的学生跨文化敏感度水平较高(4 分以上), 一半的学生跨文化敏感度水平中等偏上(3.5 4 分), 有接近 $1 / 5$ 的学 生跨文化水平偏低(3.5 分以下)。

差异认同感水平最高, 这可能由于外语专业的很多课程都会涉及到目的 语国家的概况和文化介绍。同时学生自身可以通过各种移动平台网络资源等 了解外国文化。良好的差异认同感意味着学生在进行跨文化交际时, 能更好 的理解、接受并尊重不同国家的文化差异; 为顺利进行跨文化交际奠定了基 础。表 1 数据显示, 交际信心和交际愉悦感二个维度的均值最低。这一结果 与国内一些其他研究者的调查结果一致(张雪莉 2014 [27]; 李燕 2015 [16]; 米 微、常俊跃、梁成晨 2018 [28])首先, 这二个维度对情感和行为的培养要求更

表 1. 跨文化敏感度五维度比较

\begin{tabular}{cccc}
\hline & $\mathrm{N}$ & 平均值 & 标准差 \\
\hline 跨文化敏感度 & 220 & 3.7996 & 0.33716 \\
差异认同感 & 220 & 4.1826 & 0.45428 \\
交际参与度 & 220 & 3.9182 & 0.43471 \\
交际专注度 & 220 & 3.9136 & 0.46503 \\
交际愉悦感 & 220 & 3.7576 & 0.66500 \\
交际信心 & 220 & 3.1309 & 0.56045
\end{tabular}


高, 其次, 在一定程度上也反映了国内教学中存在的问题。教师在教学中对 于鼓励学生的交际信心和提高交流意愿方面还有待提高。

\section{（二）跨文化敏感度及五维度之间的相关性分析}

为了解跨文化敏感度及五维度之间的相关性, 笔者引入了皮尔逊相关系 数进行分析, 结果如表 2 所示。

跨文化敏感度与五维度间存在显著正相关, 各个维度的提高都能促进跨 文化敏感度的提高。其中交际参与度与跨文化敏感度相关系数为 0.823 , 相关 性最高。这表明交际参与度是衡量跨文化敏感度的重要指标。五个维度的关 系中, 交际信心与交际参与度和交际愉悦感相关系数大于 0.5 达到强相关, 这说明有较强自信的学生在跨文化交际中参与感更强, 能获得更多的愉悦感; 同时学生在积极参加跨文化交流活动时, 交际愉悦感和交际信心也随之提高。 与其他研究者调查结果不同的是本研究显示差异认同感和交际信心相关性为 负值, 这可能说明并非差异认同感越强, 交际信心就越高。这意味着虽然学 生愿意接受文化差异, 但有时可能没有足够的信心在交流实践中去体验差异, 对交流中的信息接收感到担忧。

\section{（三）跨文化敏感度影响因素分析}

问卷调查中, 笔者设计了性别、四六级成绩、是否有出国经历、及外语 专业的类别四个封闭式题项。运用 SPSS 进行独立样本 $\mathrm{T}$ 检验或一次性方差分 析, 探讨四个因素分别对跨文化敏感度是否有影响, 结果显示如下。

\section{1. 性别对跨文化敏感度的影响}

有关跨文化敏感度中不同性别的影响差异, 本文采用独立样本 $\mathrm{T}$ 检验, 结果详见表 3 所示。男生 41 人, 女生 179 人, 女生的跨文化敏感度均值(3.8084)

表 2. 跨文化敏感度及五维度相关性分析

\begin{tabular}{|c|c|c|c|c|c|c|c|}
\hline & & $\begin{array}{l}\text { 跨文化 } \\
\text { 敏感度 }\end{array}$ & $\begin{array}{c}\text { 差异 } \\
\text { 认同感 }\end{array}$ & $\begin{array}{l}\text { 交际 } \\
\text { 参与度 }\end{array}$ & $\begin{array}{c}\text { 交际 } \\
\text { 专注度 }\end{array}$ & $\begin{array}{c}\text { 交际 } \\
\text { 愉悦感 }\end{array}$ & $\begin{array}{l}\text { 交际 } \\
\text { 信心 }\end{array}$ \\
\hline \multirow{2}{*}{$\begin{array}{l}\text { 跨文化 } \\
\text { 敏感度 }\end{array}$} & 皮尔逊相关性 & 1 & & & & & \\
\hline & 显著性(双尾) & & & & & & \\
\hline \multirow{2}{*}{$\begin{array}{c}\text { 差异 } \\
\text { 认同感 }\end{array}$} & 皮尔逊相关性 & $0.524^{\star *}$ & 1 & & & & \\
\hline & 显著性(双尾) & 0.000 & & & & & \\
\hline \multirow{2}{*}{$\begin{array}{c}\text { 交际 } \\
\text { 参与度 }\end{array}$} & 皮尔逊相关性 & $0.823^{\star *}$ & $0.326^{\star *}$ & 1 & & & \\
\hline & 显著性(双尾) & 0.000 & 0.000 & & & & \\
\hline \multirow{2}{*}{$\begin{array}{c}\text { 交际 } \\
\text { 专注度 }\end{array}$} & 皮尔逊相关性 & $0.556^{\star *}$ & $0.199^{\star \star}$ & $0.421^{\star *}$ & 1 & & \\
\hline & 显著性(双尾) & 0.000 & 0.003 & 0.000 & & & \\
\hline \multirow{2}{*}{$\begin{array}{c}\text { 交际 } \\
\text { 愉悦感 }\end{array}$} & 皮尔逊相关性 & $0.677^{\star *}$ & $0.144^{\star *}$ & $0.364^{\star *}$ & $0.240^{\star *}$ & 1 & \\
\hline & 显著性(双尾) & 0.000 & 0.033 & 0.000 & 0.000 & & \\
\hline \multirow{2}{*}{$\begin{array}{l}\text { 交际 } \\
\text { 信心 }\end{array}$} & 皮尔逊相关性 & $0.726^{\star *}$ & -0.015 & $0.505^{\star *}$ & $0.287^{\star *}$ & $0.589^{* *}$ & 1 \\
\hline & 显著性(双尾) & 0.000 & 0.820 & 0.000 & 0.000 & 0.000 & \\
\hline
\end{tabular}

注: **.在 0.01 级别(双尾), 相关性显著; *. 在 0.05 级别(双尾), 相关性显著。 
表 3. 性别对跨文化敏感度的影响

\begin{tabular}{ccccc}
\hline & 男生 $(\mathrm{n}=41)$ & 女生 $(\mathrm{n}=179)$ & $\mathrm{t}$ 值 & $\mathrm{P}$ 值 \\
\hline 跨文化敏感度 & 3.7612 & 3.8084 & -0.809 & 0.420 \\
差异认同感 & 3.9431 & 4.2374 & -3.202 & 0.002 \\
交际参与度 & 3.8955 & 3.9234 & -0.370 & 0.712 \\
交际专注度 & 3.9268 & 3.9106 & 0.201 & 0.841 \\
交际愉悦感 & 3.8049 & 3.7467 & 0.504 & 0.615 \\
交际信心 & 3.2293 & 3.1084 & 1.094 & 0.279 \\
\hline
\end{tabular}

略高于男生(3.7612), 但 $\mathrm{P}$ 值 $=0.420(\mathrm{P}>0.05)$ 表明男女生跨文化敏感度不存 在显著性差异。具体到各维度中, 女生的差异认同感、交际参与度均值高于 男生, 而男生的交际专注度, 交际信心, 交际愉悦感高于女生, 但五个维度 中, 只有差异认同感存在显著性差异 $\mathrm{p}=0.002(\mathrm{P}<0.05)$ 。这表明女生在尊重、 包容文化差异方面优于男生。

\section{2. 四六级成绩对跨文化敏感度的影响}

表 4 所示, 220 名被试中 89 人通过六级, 118 人通过 4 级, 13 人既没有 通过六级也没有通过四级。从结果来看, 跨文化敏感度的均值由高到低依次 是通过六级学生, 通过四级学生, 未通过考级的学生。但是 $\mathrm{P}=0.545$ 不存在 显著性差异。具体到各维度中, 四六级成绩的差异, 对跨文化敏感度的五个 维度的影响均不存在显著性差异。这一结果可能的原因为虽然学生在笔试成 绩上有差异, 比如通过了六级考试, 但是其口语水平和整体的交际能力并没 有显著提升，所以他们在交际参与度，交际信心和交际愉悦感方面并没有明 显优于成绩低的学生, 课堂观察也证实了这一点。

\section{3. 出国经历对跨文化敏感度的影响}

在出国经历方面, 表 5 的调查结果显示, 无出国经历的有 179 人, 有出 国经历的为 41 人, 有出国经历的学生在跨文化敏感度及五个维度方面的均值 均高于无出国经历的同学, 但是不存在显著性差异。这里需要指出的是由于 疫情的影响, 多数有出国经历的人仅为曾经有旅游经历或者曾经参加过夏令 营活动(时间在一个月之内), 其中仅有 5 名同学有三个月以上的访学经历, 由于样本太小, 作者没有单独分析。因此该数据只能说明短时的出国经历对 跨文化敏感度的提高无显著性影响。相关研究也认为, 跨文化敏感度难以在 短时间内发生变化(周杏英 2007 [3]; 叶敏、安然 2012 [14]; Bloom \& Miranda $2015[12])$ 。

\section{4. 外语专业的分科对跨文化敏感度的影响}

本研究调查了六个外语专业, 分别为西班牙语 60 人、德语 42 人、葡萄 牙语 33 人、意大利语 38 人、法语 14 人、俄语 22 人、捷克语 11 人(由于各 专业招收的人数不同, 研究随机选取的人数数量不等)。表 6 数据显示外语专 
表 4. 四六级成绩对跨文化敏感度的影响

\begin{tabular}{cccccc}
\hline & 六级 $(\mathrm{n}=89)$ & 四级 $(\mathrm{n}=118)$ & 没通过 $(\mathrm{n}=13)$ & $\mathrm{F}$ 值 & $\mathrm{P}$ 值 \\
\hline 跨文化敏感度 & 3.8155 & 3.7980 & 3.7051 & 0.609 & 0.545 \\
差异认同感 & 4.2285 & 4.1497 & 4.1667 & 0.769 & 0.465 \\
交际参与度 & 3.9406 & 3.9201 & 3.7473 & 1.126 & 0.326 \\
交际专注度 & 3.8989 & 3.9435 & 3.7436 & 1.159 & 0.316 \\
交际愉悦感 & 3.7640 & 3.7542 & 3.7436 & 0.008 & 0.992 \\
交际信心 & 3.1258 & 3.1441 & 3.0462 & 0.183 & 0.833 \\
\hline
\end{tabular}

表 5. 出国经历对跨文化敏感度的影响

\begin{tabular}{ccccc}
\hline & $\begin{array}{c}\text { 无出国经历 } \\
(\mathrm{n}=179)\end{array}$ & $\begin{array}{c}\text { 有出国经历 } \\
(\mathrm{n}=41)\end{array}$ & $\mathrm{t}$ 值 & $\mathrm{P}$ 值 \\
\hline 跨文化敏感度 & 3.7817 & 3.8780 & -1.658 & 0.099 \\
差异认同感 & 4.1797 & 4.1951 & -196 & 0.845 \\
交际参与度 & 3.9002 & 3.9965 & -1.281 & 0.202 \\
交际专注度 & 3.8901 & 4.0163 & -1.572 & 0.117 \\
交际愉悦感 & 3.7207 & 3.9187 & -1.728 & 0.085 \\
交际信心 & 3.1095 & 3.2244 & -1.185 & 0.237 \\
\hline
\end{tabular}

表 6. 外语专业的类别对跨文化敏感度的影响

\begin{tabular}{cccccccccc}
\hline & $\begin{array}{c}\text { 西班牙语 } \\
(\mathrm{n}=60)\end{array}$ & $\begin{array}{c}\text { 德语 } \\
(\mathrm{n}=42)\end{array}$ & $\begin{array}{c}\text { 葡萄牙 } \\
(\mathrm{n}=33)\end{array}$ & $\begin{array}{c}\text { 意大利语 } \\
(\mathrm{n}=38)\end{array}$ & $\begin{array}{c}\text { 法语 } \\
(\mathrm{n}=14)\end{array}$ & $\begin{array}{c}\text { 俄语 } \\
(\mathrm{n}=22)\end{array}$ & $\begin{array}{c}\text { 捷克语 } \\
(\mathrm{n}=11)\end{array}$ & $\mathrm{F}$ 值 & $\mathrm{P}$ 值 \\
\hline $\begin{array}{c}\text { 跨文化 } \\
\text { 敏感度 }\end{array}$ & 3.7111 & 3.8026 & 3.8245 & 3.8882 & 3.8571 & 3.8769 & 3.6629 & 1.754 & 0.110 \\
$\begin{array}{c}\text { 差异 } \\
\text { 认同感 }\end{array}$ & 4.2111 & 4.0397 & 4.1818 & 4.2588 & 4.1190 & 4.2197 & 4.3182 & 1.148 & 0.335 \\
$\begin{array}{c}\text { 交际 } \\
\text { 参与度 }\end{array}$ & 3.8238 & 3.9558 & 3.9394 & 3.9887 & 3.9286 & 4.0325 & 3.7403 & 1.275 & 0.270 \\
$\begin{array}{c}\text { 交际 } \\
\text { 专注度 }\end{array}$ & 3.8167 & 3.9365 & 3.9798 & 3.9561 & 4.1429 & 3.8485 & 3.8485 & 1.302 & 0.258 \\
$\begin{array}{c}\text { 交际 } \\
\text { 愉悦感 }\end{array}$ & 3.5889 & 3.7381 & 3.9091 & 3.8509 & 3.8509 & 3.9091 & 3.5455 & 1.510 & 0.176 \\
交际 & & & & & & & & & \\
信心 & 2.9633 & 3.2619 & 3.0909 & 3.2842 & 3.2714 & 3.2714 & 2.7273 & 3.213 & 0.005 \\
\hline
\end{tabular}

业的分科在跨文化敏感度上不存在显著性差异 $(\mathrm{P}=0.110)$, 跨文化敏感度均值 由高到低依次是意大利语、俄语、法语、葡萄牙语、德语、西班牙语、捷克 语。在五个维度方面, 交际信心维度组间存在显著性差异 $\mathrm{P}=0.005$ 。如下图 1 所示, 捷克语, 西班牙语和葡萄牙语的交际信心明显低于其他三个专业。 由于各个专业在教学过程中存在诸多不同, 只能笼统地分析, 外语专业的学 生会受所学目的语国家文化和民族性格的影响。比如意大利语专业的学生会 比较热情、开朗, 而捷克语、葡萄牙语专业更加内敛, 谨慎。已有研究证明 跨文化敏感度受到外倾性、亲和性及开放性等性格的影响(Ward \& Leong 2004 [29]; 曾斌 2009 [30])。 


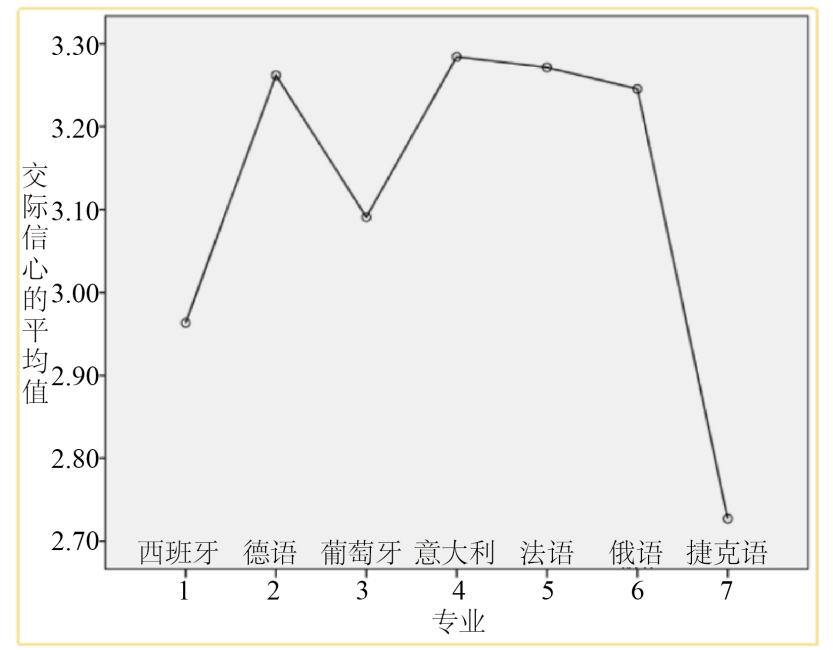

图 1. 各专业交际信心平均值图

\section{5. 总结}

本研究调查了外语专业学生的跨文化敏感度水平, 总体处于中等偏上水 平, 但还有很大的提升空间。研究发现外语专业学生跨文化敏感度中差异认 同感最强, 说明学生对异国文化的了解, 尊重方面表现较好。学生跨文化敏 感度的短板为交际信心, 这一结果与国内很多研究者的调查结果一致。这更 加证实了尊重和包容的态度是基础, 在教学中教师不能停留在对知识层面的 传授而要更注重学生 “高阶” 能力的培养, 使学生能够进行更多的跨文化 “对 话” 和反思。因此, 要想有效的提高跨文化敏感度, 提升学生的交际信心尤 为重要, 当前国内外学者对跨文化敏感度的研究趋势主要聚焦影响跨文化敏 感度的变量方面, 那么哪些变量对交际信心影响最大值得关注。

五个维度的相关分析发现, 交际信心与交际参与度和交际愉悦感强相关, 这为交际信心的提高提供了重要的依据。首先教学中教师要创造大量的跨文 化交际体验的机会, 促进课内、课外的交际实践, 如课堂上教师增设更多的 跨文化情景模拟, 分析跨文化真实案例, 引导学生分享自己的跨文化故事, 进行案例角色扮演等, 通过以上活动让学生从实际体验中体会到跨文化知识 的价值和跨文化学习的重要性。课后鼓励学生多与留学生、外教交流, 开设 文化艺术节, 举办跨文化能力大赛等, 促使他们在实际交流活动中不断积累 经验。大量的实践能够消除学生的交际焦虑, 使他们以更加妚熟的技能进行 交流。此外, 教师要重视努力营造成功的交际经验, 这对于跨文化交际能力 的培养将事半功倍。

研究还发现性别、四六级成绩、短时出国经历、外语专业类别对跨文化 敏感度不存在显著性差异。四六级成绩的数据可以推断跨文化交际能力的提 高是需要教学过程中系统、有针对性的培养的, 而并非简单的提高考试成绩 或几门课程的努力就能实现, 它需要贯穿整个教学过程多学科共同培养。短 时的旅游和夏令营活动并不能有效提高跨文化敏感度, 因此对出国留学、交 换生、海外实践等活动学校要进行精心规划, 提供相对长时(6个月以上)的国 外生活留学经历, 同时不仅要注重跨文化交流的广度还要注重跨文化交流的 
深度, 使学生的出国留学或游学真正体验跨文化学习而非简单的游览观光等。 外语专业类别说明任何语言的学习都是文化学习的体验, 教师在教学中讲授 目的语文化的同时, 要进行必要的文化对比, 使不同文化间展开有意义的对 话, 为文化发展提供开放交流进步的空间。防止学生深受目的国文化影响或 者对本国文化不自信现象, 让学生深刻全面的理解文化差异的根源, 帮助学 生形成国际视野和跨文化人格。

本研究分析了外语专业学生的跨文化敏感度现状及四个变量与跨文化敏 感度的关系，在以后的研究中可更全面的考察其他因素对跨文化敏感度的影 响, 如学生文化活动的参与、跨文化培训、长时出国经历、出国意愿、学生 口语水平、学习环境等因素。希望该调查研究结果能为我国外语专业教学培 养学生的跨文化交际能力提供有益的参考。

\section{Conflicts of Interest}

The author declares no conflicts of interest.

\section{References}

[1] 大学英语教学指南(2020 版) [M]. 北京: 教育部高等学校大学外语教学指导委员 会和高等教育出版社.

[2] King, P.M. and Baxter Magolda, M.B. (2005) A Developmental Model of Intercultural Maturity. Journal of College Student Development, 46, 571. https://doi.org/10.1353/csd.2005.0060

[3] 孙有中. 外语教育与跨文化能力培养[J]. 中国外语, 2016(3): 17-22.

[4] Chen, G. and Starosta, W. (2000) The Development and Validation of the Intercultural Sensitivity Scale. Human Communication, 3, 1-15. https://doi.org/10.1037/t61546-000

[5] Bennett, M.J. (1993) Towards Ethno Relativism: A Developmental Model of Intercultural Sensitivity. In: Paige, R.M., Ed., Education for the Intercultural Experience, Intercultural Press, Yarmouth.

[6] Hammer, M.R. and Bennett, M.J. (2002) The Intercultural Development Inventory (IDI) Manual. Intercultural Communication Institute, Portland.

[7] Hammer, M.R., Bennett, M.J. and Wiseman, R. (2003) Measuring Intercultural Sensitivity: The Intercultural Development Inventory. International Journal of Intercultural Relation, 27, 421-444. https://doi.org/10.1016/S0147-1767(03)00032-4

[8] Straffon, D.A. (2003) Assessing the Intercultural Sensitivity of High School Students Attending an International School. International Journal of Intercultural Relations, 27, 487-501. https://doi.org/10.1016/S0147-1767(03)00035-X

[9] Holma, K., Nokelainenb, P. and Tirri, K. (2009) Relationship of Gender and Academic Achievement to Finnish Students' Intercultural Sensitivity. High Ability Studies, 20, 187-200. https://doi.org/10.1080/13598130903358543

[10] Tamama, E. and Krauss, S.E. (2017) Ethnic-Related Diversity Engagement Differences in Intercultural Sensitivity among Malaysian Undergraduate Students. International Journal of Adolescence and Youth, 22, 137-150. https://doi.org/10.1080/02673843.2014.881295

[11] Frit, W., Möllenberg, A. and Chen, G.M. (2002) Measuring Intercultural Sensitivity in Different Cultural Context. Intercultural Communication Studies, 2, 165-176. 
[12] Bloom, M. and Arturo Miranda, A. (2015) Intercultural Sensitivity through Short-Term Study Abroad. Language and Intercultural Communication, 15, 567-580. https://doi.org/10.1080/14708477.2015.1056795

[13] Kim, J., Choi, J. and Tatar, B. (2017) English-Medium Instruction and Intercultural Sensitivity: A Korean Case Study. Journal of Studies in International Education, 21, 467-482. https://doi.org/10.1177/1028315317720767

[14] 周杏英. 大学生跨文化敏感水平测试[J]. 山东外语教学, 2007(5): 62-66.

[15] 邵思源, 陈坚林. 一项对高中英语教师跨文化交际敏感度的调查[J]. 外语学刊, 2011(3): 144-147.

[16] 李燕. 北京市大学生跨文化敏感度调查研究——以 $N$ 大学为例 $[J]$. 教育理论与实 践, 2015(18): 13-15.

[17] 崔海英, 王静. 应用型高校“多维度”跨文化交际能力培养模式的构建 [J]. 河北科 技师范学院学报, 2018, 17(3): 79-85.

[18] 彭世勇. 跨文化敏感: 英语专业与非英语专业学生对比 [J]. 宁夏大学学报: 人文 社会科学版, 2007(1): 171-176.

[19] 王晓玲. 跨文化敏感: 民族院校与非民族院校学生对比研究 [J]. 北方民族大学学 报(哲学社会科学版), 2012(1): 73-77.

[20] 贾曼丽. 高职生跨文化敏感与跨文化效力的相关性研究 [J]. 外国语文, 2014, 30(3): 171-174.

[21] 张芝花. 跨文化敏感度与英语文化负载词汇习得的相关性研究 [J]. 外语教学, 2015, 36(3): 60-63.

[22] 赵萱. 基于 DMIS 模式的留学生跨文化敏感培养研究[J]. 内蒙古师范大学学报 (教育科学版), 2011(7): 96-98.

[23] 蒋璜. 在外语教学中运用 DMIS 模式提高学生跨文化能力[J]. 信息化建设, 2016(5): 184-186.

[24] 马黎. 高等农业院校大学生跨文化敏感度实证调查分析 [J]. 沈阳农业大学学报: 社会科学版, 2017(3): 335-339.

[25] 张红玲. 以跨文化教育为导向的外语教学: 历史、现状与未来 [J]. 外语界, 2012(2): 2-7.

[26] 沈兴涛. 一项针对英语专业学生跨文化敏感度的实证研究 [D]: [硕士学位论文]. 上海: 上海外国语大学, 2012.

[27] 张雪莉. 中外合作院校英语专业大学生跨文化敏感度调查[J]. 山西师大学报(社 会科学版), 2014(3): 158-160.

[28] 米微, 常俊跃, 梁成晨. 国别社会文化系列课程教学背景下英语专业学生跨文化 敏感度现状分析[J]. 语言教育, 2018, 6(1): 12-17.

[29] Ward, C., Leong, C.H. and Low, M. (2004) Personality and Sojourner Adjustment: An Exploration of the Big Five and the Cultural Fit Proposition. Journal of Cross-Cultural Psychology, 2, 137-151. https://doi.org/10.1177/0022022103260719

[30] 曾斌. 中国人跨文化敏感性现状及影响因素研究[D]: [硕士学位论文]. 上海: 华 东师范大学, 2009 . 


\section{Appendix (Abstract and Keywords in Chinese)}

\section{外语专业学生跨文化敏感度及影响因素实证研究}

摘要: 本研究采用陈国明和 Starosta 研发的跨文化敏感度量表, 对某高 校外语专业 220 名学生的跨文化敏感度进行了调查。调查发现外语专业学生 的跨文化敏感度处于中等偏上水平。五项维度中差异认同感最强、交际参与 度位居第二、交际专注度和交际愉悦感依次排后，而交际信心最弱；通过皮 尔逊相关分析显示五维度的关系中交际信心、交际参与度和交际愉悦感强相 关。研究还调查了性别、四六级成绩、出国经历及外语专业类别对跨文化敏 感度的影响。结果显示, 男女生的跨文化敏感度不存在显著性差异, 但在差 异认同感维度上差异显著; 四六级成绩及短时出国经历对跨文化敏感度不存 在显著性差异; 外语专业的类别对跨文化敏感度不存在显著性差异，但是在 交际信心维度上组间存在显著性差异。

关键词: 外语专业学生, 跨文化敏感度, 影响因素 